\title{
HERBIG-HARO OBJECTS AS SEARCHLIGHTS FOR DENSE CLOUD CHEMISTRY
}

\author{
S. D. TAYLOR \\ University College London, Gower St., \\ London WC1E 6BT, England
}

\section{Introduction}

Stars form from dense pockets of gas that have undergone gravitational collapse, and young stars are still embedded in this molecular material. There is no lack of evidence that the outflows from these stars, manifested in the form of Herbig-Haro objects and jets, interact dynamically with the dense gas either in the form of shock excitation or the imparting of momentum; molecular outflows, $\mathrm{H}_{2}$ emission, and perhaps also $\mathrm{CO}$ and $\mathrm{SiO}$ bullets and jets, are tracers of this phenomenon. However there are a set of radio observations of molecules showing emission near $\mathrm{HH}$ objects that are remarkable for their apparent lack of signatures of such dynamic interaction. A likely cause of this 'quiescent' emission is a radiative interaction between the $\mathrm{HH}$ shock system emission and ambient gas.

The 'cores' from which stars form are localised $(\sim 0.1 \mathrm{pc})$ density enhancements within the molecular clouds traced by the $\mathrm{CO}$ molecule, and there is a great deal of interest in their chemistry since many molecules, some rather complex, have been observed in them (Williams 1994). These molecules are able to form because they are shielded from the ambient interstellar radiation field, but a number of uncertainties remain, amongst them the effect of accretion of gas molecules onto the surfaces of dust grains to form ice mantles, and the possibility of a subsequent surface chemistry. These ices are observed in infrared absorption in a number of molecules $\left(\mathrm{H}_{2} \mathrm{O}, \mathrm{CO}, \mathrm{CH}_{3} \mathrm{OH}, \mathrm{CH}_{4}, \mathrm{CO}_{2}\right.$; Whittet et al. 1996) when suitable background sources are present, but must exist to some extent in all gas exposed to a greatly attenuated UV flux.

In this contribution I will describe a model in which $\mathrm{HH}$ jets propagate into a clumpy medium, and each of these clumps contains dust that can accrete ice mantles. If a clump finds itself in the vicinity of the jet it may be 
exposed to radiation from one of its working surfaces, and if this can remove the mantles the ensuing gas phase chemistry can produce large abundance enhancements in many molecules, perhaps rendering them observable. I will then discuss the nature of these clumps, and how the illumination of the clumps by the jet 'searchlight' can help us to understand the dust:gas interaction in dense regions.

\section{Observations of Quiescent Emission}

Cores are detected by means of molecular transitions that trace gas denser than that of the ambient cloud, $\left(n_{H} \geq 10^{4} \mathrm{~cm}^{-3}\right.$ against $n_{H} \sim 10^{3} \mathrm{~cm}^{-3}$, $n_{H}$ is the number of hydrogen nuclei per unit volume). In order to study dense gas around $\mathrm{HH}$ objects it is also necessary to have high angular resolution to determine the position relative to the shocks, and so such observations have tended to be made using interferometers, although submm single dish observations can also achieve high resolution. For these reasons the $\mathrm{HCO}^{+}(\mathrm{J}=1-0)$ and $\mathrm{NH}_{3}(1,1)$ and $(2,2)$ inversion transitions have mostly been used. Rudolph and Welch $(1988,1992)$ mapped HH7-11 and $\mathrm{HH} 34$ in $\mathrm{HCO}^{+}$, and Rudolph (1992) also looked at the jet in L1551 in the same molecule, all using the Hat Creek interferometer. $\mathrm{HH} 1$ and $\mathrm{HH} 2$ were imaged by Torrelles et al. $(1992,1993)$ in ammonia using the VLA, as was HH80 (North) by Girart et al. (1994). In addition Davis, Dent and Bell Burnell (1990) observed $\mathrm{HCO}^{+}(\mathrm{J}=4-3)$ near $\mathrm{HH} 1$ and $\mathrm{HH} 2$ using JCMT and Davis \& Dent (1993) found $\mathrm{NH}_{3}$ in $\mathrm{HH} 34$ with the Effelsberg telescope. All of these observations share the following characteristics;

(i) The emission is confined to patches of emission close to, but downwind of, the optical shock emission. These localised areas of emission, which we term clumps to distinguish from the core emission that surrounds the source of the outflow, have sizes of $\sim 10^{\prime \prime}-20^{\prime \prime}$, which is typically $\sim 0.02 \mathrm{pc}$.

(ii) The clumps appear dynamically unaffected, in the sense that the lines have widths of $<1 \mathrm{~km} \mathrm{~s}^{-1}$, and centres within $1-2 \mathrm{~km} \mathrm{~s}^{-1}$ of the core emission surrounding the outflow source.

At this conference, Dent (1997) has presented further observations around $\mathrm{HH} 2$ using $\mathrm{JCMT}$, and detected $\mathrm{HCO}^{+}(\mathrm{J}=3-2$ and $\mathrm{J}=4-3)$, but also found weak emission from the new molecules $\mathrm{H}_{2} \mathrm{CO}\left(5_{15}-4_{14}\right), \mathrm{N}_{2} \mathrm{H}^{+}(\mathrm{J}=4-3)$, and $\mathrm{HCS}^{+}(\mathrm{J}=8-7)$, together with the non-detection of transitions from several other molecules. Once again the $\mathrm{HCO}^{+}$shares the above characteristics. 


\section{Possible Explanations}

It is necessary to explain not only why these clumps appear ahead of $\mathrm{HH}$ objects, but also why they do not appear elsewhere. There are three ways in which this may be possible;

(i) Coincidental alignment. It could be reasoned that with a small sample set it may be that clumps dense enough to emit are found by chance projected where they are. The apparently high detection rate combined with the general absence of emission elsewhere in the observed fields would seem to argue against this, as does the presence of clumps ahead of more than one shock system in the same source. For example this emission is found ahead of each of the optical knots HH1 and HH2, and ahead of each of the knots in HH7-11 except for HH7.

(ii) Shocked cloudlet. These observations initially seem to favour the 'shocked cloudlet' hypothesis for the origin of $\mathrm{HH}$ objects themselves, whereby a stellar wind strikes a density enhancement in the cloud causing a bow shock around it and a shock within it. Reasons given in the past for why this model is not generally applicable are given added weight with the addition of this radio emission, even though in theory the presence of a shock could lead to extra molecular emission compared with other ambient clumps either by chemical enhancement or an increase in density on cooling. To explain the generally high proper motions of $\mathrm{HH}$ objects (e.g. HH1-2) the cloudlet must be shocked and accelerated without being destroyed, but even if this is so the clumps seen in molecular emission have ambient cloud velocities. Whilst it is possible to explain the proper motion by invoking the fragmentation of the cloudlet, the radio emission is more difficult to explain since rotational temperatures derived from the ammonia transitions suggest the gas is cold, and if this is cooled postshock gas the shock velocity in the cloudlet must be very small for the gas to have a line centre so close to that of the ambient gas. Furthermore, although in HH7-11 the $\mathrm{HCO}^{+}$emission is very close to the optical emission, in other objects it is clearly spatially separated, and in the case of HH34 the optical arc structure points in the wrong direction and appears morphologically distinct from the radio.

(iii) Irradiated clump. Given the quiescent nature of the emission, an attractive alternative is that of an ambient clump that has been unaffected dynamically by the outflow, but happens to lie sufficiently close to the shock system that it is subject to the radiation field it generates, which somehow enhances the emission from certain molecules. This scenario was proposed by Wolfire and Königl (1993, WK93) specifically to model the $\mathrm{HCO}^{+}$observations. In that paper the $\mathrm{HCO}^{+}(\mathrm{J}=1-0)$ line is enhanced both by an increase in fractional abundance of the molecule $x\left(\mathrm{HCO}^{+}\right)\left(=n\left(\mathrm{HCO}^{+}\right) / n_{H}\right)$ 
due to chemical effects, and an increase in excitation because of electron collisions in the irradiated gas which has a relatively high fractional ionisation. Both effects are the consequence of the impact of high energy photons (X-rays and EUV) from a strong shock on a thin layer at the edge of the clump (visual extinction $\mathrm{A}_{V}<0.1$ magnitudes). Photoionisation of $\mathrm{H}_{2}$ (which is by far the dominant gas phase species) heats and partially ionises this layer and allows molecules like $\mathrm{OH}$ and $\mathrm{H}_{2} \mathrm{O}$ to form quickly by the endothermic neutral-neutral reactions that are well known from work on shock chemistry, or by the ion-molecule route thought to occur in low temperature interstellar clouds but enhanced by the high abundance of $\mathrm{H}^{+}$. Ordinarily any molecular species would be quickly destroyed in this harsh environment, but the emission from a shock is comprised mostly of line photons that do not overlap the dissociation bands of $\mathrm{H}_{2}$ and $\mathrm{CO}$, and as a consequence they retain their 'pre-shock radiation' high abundances for some time after that radiation is switched on and can contribute to the chemistry. $\mathrm{C}^{+}$still becomes very abundant as a result of the dissociation of $\mathrm{CO}$ molecules, and it is its reaction with $\mathrm{OH}$ and $\mathrm{H}_{2} \mathrm{O}$ that produces $\mathrm{HCO}^{+}$,

$$
\begin{gathered}
\mathrm{C}^{+}+\mathrm{H}_{2} \mathrm{O} \rightarrow \mathrm{HCO}^{+}+\mathrm{H} \\
\mathrm{C}^{+}+\mathrm{OH} \rightarrow \mathrm{CO}^{+} \stackrel{\mathrm{H}_{2}}{\longrightarrow} \mathrm{HCO}^{+} .
\end{gathered}
$$

Whilst this model is of relevance to objects like HH1-2 where there are clearly very fast shocks capable of producing high energy radiation, it is questionable how valid it is for 'low excitation objects' (LEO's; Böhm \& Solf 1992) such as HH7-11, where the shock velocities appear to be no more than $30-40 \mathrm{~km} \mathrm{~s}^{-1}$, although the linewidths are appreciably larger. HH34 appears to be an intermediate case, requiring shock velocities of $\sim 120-170 \mathrm{~km} \mathrm{~s}^{-1}$. It is also not clear how the subsequent observations of ammonia can be similarly explained, since an enhancement over other ambient clump abundances is required. The temperatures even in the very edge of the clump are unlikely to be high enough to drive neutral-neutral formation due to the high barrier $\left(\Delta \mathrm{E}=1.6 \times 10^{4} \mathrm{~K}\right)$ of the initiating reaction

$$
\mathrm{N}+\mathrm{H}_{2} \rightarrow \mathrm{NH}+\mathrm{H},
$$

and formation via nitrogen ions is unlikely to produce more $\mathrm{NH}_{3}$ than in cold gas since the barriers in the reaction chain do not seriously affect the final abundance of $\mathrm{NH}_{3}$.

We have considered an alternative model, which is in fact simply a variation on the WK93 model, but where the shock radiation field is considered 
to have negligible strength above $13.6 \mathrm{eV}$. The key addition in this model is the inclusion of grain surface processes.

\section{Alternative Model}

The processes that result in the collapse of a cloud to form a star-forming core appear likely to result in fragmentation, and the pieces not massive enough to go on to form stars would seem to be the most likely source of the clumps presumed here to be exposed to the shock radiation. It is the presence of clumps such as these that we have invoked previously as the reason why CS $(\mathrm{J}=1-0)$ emission is seen quite generally to have a larger spatial extent than $\mathrm{NH}_{3}(1,1)$ in star-forming regions, despite the CS transition having a higher critical density and therefore supposedly tracing denser gas (Taylor, Morata and Williams 1996). This is based on the different formation timescales for the two molecules as the clumps form from more diffuse gas. These clumps would exist within a relatively tenuous interclump medium that surrounds the star-forming core, and through which the stellar outflows are propagating (in this picture young outflows such as HH211 seen only in the infrared would be expected to show molecular emission at their heads since they are still embedded in the high density core).

The interclump medium will, to some extent, allow through the interstellar radiation field, and this means that the clumps are exposed to a UV radiation field at some level even in the absence of shocks. However, beyond a certain value of visual extinction the dust in the interior will accrete ice mantles, like those seen in absorption in the infrared towards suitable background sources. The mantles are likely to be hydrogenated molecules of abundant atomic species, such as $\mathrm{H}_{2} \mathrm{O}, \mathrm{NH}_{3}, \mathrm{CH}_{4} .$. , and although species such as methanol and carbon dioxide have also been seen it is possible that they only occur in the densest cores where accretion of volatile $\mathrm{CO}$ can occur followed by surface reaction or irradiation by a protostar. If the $\mathrm{HH}$ shock radiation can remove these mantles then a chemistry can follow that will enhance the abundance of many molecules, $\mathrm{HCO}^{+}$and $\mathrm{NH}_{3}$ included.

To this end, we have modelled such a clump as a 1D slab of constant density and temperature exposed to a reduced flux of UV photons $\chi_{0}$ in units of the Draine interstellar radiation field (Draine 1978). This is then similar to a model of a photodissociation region (e.g Hollenbach, Takahashi,\& Tielens 1991). To obtain the clump initial chemical conditions just prior to the arrival of the $\mathrm{HH}$ object we use a network of chemical equations and associated reaction rates and numerically determine the fractional abundance of each species as a function of time, starting with everything atomic apart from molecular hydrogen (consistent with collapse from a diffuse state). Freezeout of species onto dust surfaces is included, the only surface chemistry 
presumed being the hydrogenation of atoms to saturation. The chemistry is halted before complete freeze-out occurs, in line with observations that suggest that less than half of the observed gas phase abundance is seen in solid form (Chiar et al. 1995). The time is reset to $t=0$, and the shock radiation is switched on, and at the same time complete mantle release is assumed. The strength of this radiation field depends on the shock velocity and the preshock (interclump) density and the nature of it was examined in some detail by Wolfire and Königl (1991). They converted the spectrum into an integrated UV flux in units of the interstellar flux (here $\chi_{1}$ ), only a component of which is continuum that can dissociate $\mathrm{H}_{2}$ or $\mathrm{CO}\left(\chi_{c}\right)$. In our models we take values of $\chi_{1}$ and $\chi_{c}$ similar to those of WK93, for the sake of comparison. We then once again follow the time-dependent chemistry.

The results for which the clump has a density of $n_{H}=5 \times 10^{4} \mathrm{~cm}^{-3}$, temperature $\mathrm{T}=10 \mathrm{~K}$, and $\chi_{1}=20, \chi_{c}=2$, are shown in Figure 1. Here the fractional abundances of a number of chemical species are plotted as a function of visual extinction for four different times after the shock radiation is switched on. As the mantles are released the observed molecules (dashed lines) briefly attain high abundances before a dissociation wave traverses the clump. The largest possible abundances in the absence of additional radiation are achieved in the absence of freezeout, and for $\mathrm{NH}_{3}$ and $\mathrm{HCO}^{+}$these peak at $1 \times 10^{-8}$ and $2 \times 10^{-10}$ respectively. There is a clear enhancement in the model abundances by orders of magnitude, that might explain the observed emission. It is obvious that $\mathrm{NH}_{3}$ is produced directly by mantle desorption, remaining at its initial fraction until destroyed by UV photons. $\mathrm{HCO}^{+}$is produced by reaction (1), however now the $\mathrm{H}_{2} \mathrm{O}$ is provided by mantle chemistry rather than gas phase reactions. Since for $\mathrm{A}_{V} \gtrsim_{1}$ and $\mathrm{t} \lesssim 20 \mathrm{yr}$ the $\mathrm{C}^{+}$is formed mostly by the preshock $\chi_{0} \mathrm{UV}$ field a clump more shielded than we have assumed will have a lower $\mathrm{HCO}^{+}$abundance at these points. Models with $\mathrm{C}^{+}$formed entirely from $\mathrm{CH}_{4}$ released from mantles (i.e due to the $\chi_{1} \mathrm{UV}$ field) show $\mathrm{HCO}^{+}$down by factors of 2-3. For $A_{V} \lesssim 1$ or $\mathrm{t} \gtrsim_{20}$ yr there is little difference.

Except at the very edge of the clump the $\mathrm{HCO}^{+}$is also higher than that produced in the WK models, and this difference increases if the temperature in the clump is increased (see Taylor \& Williams 1996) since the destruction of $\mathrm{HCO}^{+}$by dissociative recombination has a steep inverse temperature dependence,

$$
\mathrm{HCO}^{+}+\mathrm{e}^{-} \rightarrow \mathrm{CO}+\mathrm{H}, \quad k \propto T^{-1} .
$$

A simple comparison of the heating rate due to photoelectric ejection from dust (Bakes \& Tielens 1994) with cooling due to CO, CII and OI (Hollenbach \& McKee 1979), however, suggests that $\mathrm{T}>30 \mathrm{~K}$ is unlikely except in 

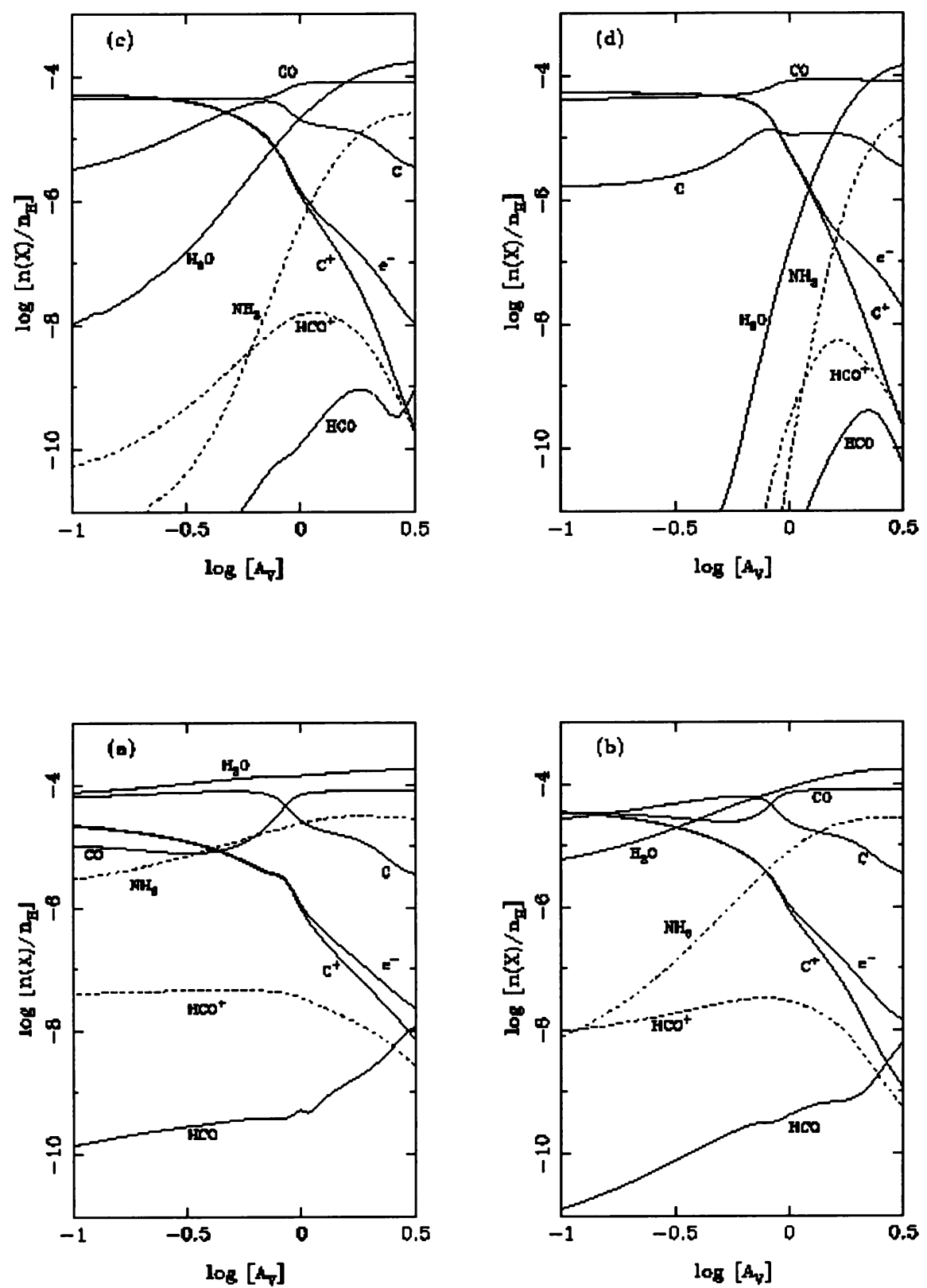

Figure 1. Fractional abundances (relative to hydrogen nuclei) of various species as a function of visual extinction (magnitudes) into a clump, for times $3,10,30$ and 100 (a, $b, c, d)$ years after the shock radiation is turned on. 
the clump edge, as can be inferred from the thermal balance calculations in WK93.

The UV radiation field destroys molecules in cold gas and makes the chemistry very time-dependent. Lower radiation fields have the effect simply of extending the chemical timescales, hence these results will continue to hold for LEO's. The fact that the molecules shown in Fig.1 are destroyed on timescales of tens of years would imply that this chemistry may be progressing on observable timescales, a unique feature of these objects.

Other molecules are also enhanced in these models over those that would be obtained in a quiescent clump. The molecules we include in the chemistry are fairly simple since we have included relatively few species $(\sim 100)$ composed of the elements $\mathrm{H}, \mathrm{N}, \mathrm{C}, \mathrm{O}, \mathrm{S}$, and $\mathrm{Mg}$, and more complicated species would have to be included to properly analyse what the observational signatures might be. Photodissociation products of desorbed molecules have high abundances, such as $\mathrm{CH}_{2}, \mathrm{CH}, \mathrm{OH}, \mathrm{NH}_{2}$, but only the last two seem to have the potential to be observed in radio emission at reasonable angular resolution. Other potential species are derived from these products, for example CN and NO. Sulphur containing species, such as $\mathrm{HCS}^{+}$which is formed in a similar way to $\mathrm{HCO}^{+}\left(\mathrm{C}^{+}\right.$reacting with $\left.\mathrm{H}_{2} \mathrm{~S}\right)$ may also be abundant if the depletion of sulphur into refractory grain material is not too high. It may be that grain surface reactions involving hydrogenation of accreted $\mathrm{CO}$ also occur, in which case formaldehyde (tentatively detected by Dent) and methanol may also be observable.

\section{Discussion Points}

\subsection{CLUMPS IN MOLECULAR OUTFLOWS}

Dynamically unaffected clumps are an attractive proposition if they lie ahead of the leading jet bowshock, however this is clearly not the case in HH7-11, and what is more it appears that the shocks at HH1, HH2 or HH34 also do not represent the end of the jet (Reipurth \& Heathcote 1992, Ogura 1996). $\mathrm{HH}$ objects embedded in the flow from an earlier jet episode may partly account for the fact that shock velocities inferred by excitation analysis are often much lower than those from linewidths or proper motion measurements. It seems likely that the clumps, whilst not lying in the path of the jet as would be the case in the shocked cloudlet hypothesis, are at least embedded in a lower velocity molecular outflow caused by the interaction of the jet with the ambient medium. The molecular outflows in HH1-2 and HH34 are weak (Chernin \& Masson 1995), but a molecular outflow is observed in HH7-11 even at velocities $>100 \mathrm{~km} \mathrm{~s}^{-1}$, though this material sits on the optical axis and the clumps are more likely to find themselves in outflows with more standard velocities. Since this outflow 
has been traced in CO ( $\mathrm{J}=1-0)$ (Liseau et al. 1988), which emits at low gas densities, and since the other outflows are so weak, we favour the view that this material is of low enough density to leave the clumps essentially unaffected, provided that entrainment of clump material in the boundary layers along the sides of the clump does not destroy the clumps. On the other hand clumps less stable will be shocked and swept up, and may be partly responsible for the $\mathrm{HCO}^{+}(\mathrm{J}=4-3)$ observations of $\mathrm{HH} 7-11$ by Dent et al. (1993).

\subsection{MANTLE ACCRETION EFFICIENCY}

Grain surfaces accrete gas phase species at the same efficiency regardless of depth in our models, which is clearly not applicable as $\mathrm{A}_{V}$ decreases, since solid state IR absorption features are not seen below a (region dependent) critical extinction. In fact this is possibly a consequence of the general interstellar IR radiation field preventing the formation of a surface monolayer (Hartquist, Williams \& Whittet 1992). We have also assumed that the shock radiation field can instantly remove these mantles, which probably requires non-thermal photodesorption since thermal evaporation requires significant grain heating, and the grain temperature has only a weak dependence on the UV flux (Hollenbach et al. 1991). The sensitivity of the surface abundances to the accretion and desorption rates is an issue that requires further investigation.

An objection may be raised that we have postulated the release of ammonia from ice mantles, yet to date no ammonia has been observed in the solid phase. Whilst the reason why the hydrogenation of nitrogen atoms on the surface isn't as efficient as might be expected is puzzling, the current upper limits of a few percent of solid water still allow for a surface fractional abundance orders of magnitude greater than that formed from purely gas phase processes, hence our results can still be consistent with the observations.

\subsection{HOT CORE ANALOGY}

The irradiation model presented here bears many similarities with models of hot cores in high mass star forming regions. There the onset of stellar radiation following the birth of a new star is thought to remove mantles and start a chemistry that actually produces some of the most complex species known in space (Millar 1993). It is an intriguing possibility that some of these molecules may be observable in the low mass star forming regions where most of these $\mathrm{HH}$ objects are formed. A note of caution must be added though, for although the hot cores and the clumps are comparable in size they are probably considerably denser $\left(\mathrm{n}_{H} \sim 10^{7} \mathrm{~cm}^{-3}\right)$ so that the 
column densities of a given species are likely to be much greater, and the surface chemistry may be more complex. It may also be that some of the chemistry that occurs in the hot cores is a consequence of the inferred gas temperature of $\sim 200 \mathrm{~K}$, which is greater than that possible in clump interiors.

Deuteration fractionation is possible in these cold clumps through purely gas phase chemistry, but Brown \& Millar (1989) showed that hydrogenation or deuteration on grains can lead to much larger abundances on the surface. Since a major assumption in our model is that large quantities of (undetectable) water are released into the gas phase, a good test could be a search for HDO, which does have detectable transiticns in cold clouds.

\section{References}

Bakes,E.L.O,Tielens,A.G.G.M.,1994, $A p J, 427,822$

Böhm,K.H,Solf,J.,1992, $A J, 104,1193$

Brown,P.D.,Millar,T.J.,1989,MNRAS,237,661

Chiar,J.E.,Adamson,A.J.,Kerr,T.H.,Whittet,D.C.B.,1995, ApJ,455,234

Chernin,L.M.,Masson,C.R.,1995, ApJ,443,181

Davis,C.J.,Dent,W.R.F.,1993,MNRAS,261,371

Davis,C.J.,Dent,W.R.F.,Bell Burnell,S.J.,1990,MNRAS,244,173

Dent, W.R.F.,1997, in Low Mass Star Formation - from Infall to Outflow, poster proceedings of IAU Symp. No. 182, ed. F. Malbet \& A. Castets, Grenoble, p.88

Dent,W.R.F., et al. ,1993,MNRAS,262,L13

Draine,B.T.,1978, $A p J S, 36,595$

Girart,J.M., et al. ,1994,ApJ,435,L145

Hartquist, Williams \& Whittet 1992 MNRAS,258,599

Hollenbach,D.J.,McKee,C.F.,1979, ApJS,41,555

Hollenbach,D.J.,Takahashi,T.,Tielens,A.G.G.M.,1991, ApJ,377,192

Liseau,R.,Sandell,G.,Knee,L.B.G.,1988, $A \& A, 192,153$

Millar,T.J.,1993,Dust and Chemistry in Astronomy. Millar,T.J.,Williams,D.A. (eds.),IOP Publishing Ltd., Bristol.

Ogura,K.,1996, ApJ,450,L23

Reipurth,B.,Heathcote,S.,1992, $A \& A, 257,693$

Rudolph,A.,1992,ApJ,397,L111

Rudolph,A.,W.J.,Welch,1988, $A p J, 326, \mathrm{~L} 31$

Rudolph,A.,W.J.,Welch,1992, $A p J, 395,488$

Taylor,S.D.,Morata,O.,Williams,D.A., $A \& A, \mathbf{3 1 3}, 269$

Taylor,S.D.,Williams,D.A.,1996,MNRAS,282,1343

Torrelles,J.M., et al. ,1992,ApJ,396,L95

Torrelles,J.M., et al. ,1993, ApJ,417,655

Whittet,D.C.B., et al. ,1996, $A \& A, \mathbf{B 1 5}, \mathrm{L} 357$

Williams,D.A.,1994, Contemporary Physics,35,269

Wolfire,M.G.,Königl,A.,1991, $A p J, 383,205$

Wolfire,M.G.,Königl,A.,1993,ApJ,415,204 (WK93) 